\title{
THE CONGRUENCE LATTICE OF A COMBINATORIAL STRICT INVERSE SEMIGROUP
}

\author{
by KARL AUINGER
}

(Received 27th February 1992)

The congruence lattice of a combinatorial strict inverse semigroup is shown to be isomorphic to a complete subdirect product of congruence lattices of semilattices preserving pseudocomplements.

1991 Mathematics subject classification. 20M10, 08A30.

\section{Introduction}

Congruence lattices of semilattices admit several remarkable properties. Let $\mathscr{S}$ denote the class of all semilattices, let $Y \in \mathscr{S}$ and $\mathscr{C}(Y)$ be the congruence lattice of $Y$. By Papert [11], $\mathscr{C}(Y)$ is completely (meet-)semidistributive, (weakly) relatively pseudocomplemented and thus also pseudocomplemented, and each non universal congruence $\rho \in \mathscr{C}(Y)$ is the intersection of coatoms (which are also characterized). Hall [5] proved that $\mathscr{C}(Y)$ is semimodular (and thus satisfies the Jordan-Dedekind chain condition), and described the atoms of $\mathscr{C}(Y)$. Jones [9] has shown that $\mathscr{C}(Y)$ is $M$-symmetric. Freese and Nation [2] proved that the class $\mathscr{C}(\mathscr{S})=\{\mathscr{C}(Y) \mid Y \in \mathscr{S}\}$ of all congruence lattices of semilattices does not satisfy any non trivial lattice identity. Finally, Zhitomirskij [13] gave a characterization of all lattices which are isomorphic to congruence lattices of semilattices. Quite recently, Freese and Nation [3] have again considered congruence lattices of finite semilattices from a more lattice theoretic point of view. They have shown, for instance, that the congruence lattice of a finite semilattice (in fact, of a finite combinatorial inverse semigroup) is upper bounded (which is stronger than meet-semidistributivity).

A combinatorial strict inverse semigroup $S$ is an (inverse) subdirect product of combinatorial Brandt semigroups (and possibly the trivial group) (see Petrich [12]). This class forms a variety of inverse semigroups and will be denoted by $\mathscr{B}$. It is well known that $\mathscr{B}$ is the unique combinatorial cover of $\mathscr{S}$ in the lattice of inverse semigroup varieties [12]. The purpose of this note is to establish several of the above mentioned properties for congruence lattices of combinatorial strict inverse semigroups. Let $T \in \mathscr{B}$. We shall describe atoms and coatoms in $\mathscr{C}(T)$ and show that each non universal congruence $\rho \in \mathscr{C}(T)$ is the intersection of coatoms. Further, we shall prove that $\mathscr{C}(T)$ is a complete subdirect product of congruence lattices of semilattices. We infer that each lattice implication which holds $\mathscr{C}(\mathscr{S})$ also holds in $\mathscr{C}(\mathscr{B})=\{\mathscr{C}(T) \mid T \in \mathscr{B}\}$. In 
particular, $\mathscr{C}(T)$ is completely semidistributive. In addition, $\mathscr{C}(T)$ is $M$-symmetric (which in fact has been already shown by Jones [9]). Furthermore, it is proved that the mentioned subdirect decomposition of $\mathscr{C}(T)$ preserves (weak) relative pseudocomplements and hence $\mathscr{C}(T)$ is pseudocomplemented for any $T \in \mathscr{B}$. Finally, an example shows that the classes $\mathscr{C}(\mathscr{S})$ and $\mathscr{C}(\mathscr{B})$ do not coincide.

\section{Preliminaries: combinatorial strict inverse semigroups}

For undefined notions, the reader is referred to the books of Howie [7] (general semigroups), Petrich [12] (inverse semigroups) and Grätzer [4] (lattices). For an arbitrary semigroup $S$ let $\mathscr{C}(S)$ denote the (complete) lattice of all congruences on $S$. Join and meet are denoted by $\vee$ and $\cap$ (or $V$ and $\bigcap$ ). The lattice of all equivalence relations on a set $X$ is denoted by $\mathscr{E}(X)$. The identical and universal relations on the set $X$ are denoted by $\varepsilon=\varepsilon_{X}$ and $\omega=\omega_{X}$, respectively.

Combinatorial strict inverse semigroups admit the following description (see Petrich [12, XIV] or Nambooripad [10]).

Theorem 2.1. Let $X$ be a partially ordered set, for each $\alpha \in X$ let $I_{a}^{*}$ be the non zero part of a combinatorial Brandt semigroup $I_{\alpha}$ such that $I_{a}^{*} \cap I_{\beta}^{*}=\emptyset$ whenever $\alpha \neq \beta$. For $\alpha \geqq \beta$ let $f_{\alpha, \beta}: I_{\alpha}^{*} \rightarrow I_{\beta}^{*}$ be a partial homomorphism subject to the following conditions

(1) $f_{\alpha, \alpha}=\mathrm{id}_{l_{q}}$ for all $\alpha \in X$,

(2) $f_{\alpha, \beta} f_{\beta, \gamma}=f_{\alpha, \gamma}$ whenever $\alpha \geqq \beta \geqq \gamma$,

(3) for any $x \in I_{\alpha}^{*}, y \in I_{\beta}^{*}, \alpha, \beta \in X$, the set

$$
D(x, y)=\left\{\gamma \leqq \alpha, \beta \mid\left(x f_{\alpha, y}\right)\left(y f_{\beta, y}\right) \neq 0 \text { in } I_{y}\right\}
$$

has a greatest element, to be denoted by $\delta(x, y)$.

Put $S=\bigcup_{\alpha \in X} I_{\alpha}^{*}$ and define a product on $S$ by

$$
x y=\left(x f_{\alpha, \delta(x, y)}\right)\left(y f_{\beta, \delta(x, y)}\right)
$$

where $x \in I_{\alpha}^{*}, y \in I_{\beta}^{*}$. Then the groupoid $S$, to be denoted by $\left(X ; I_{\alpha}, f_{a, \beta}\right)$ is a combinatorial strict inverse semigroup. Conversely, every combinatorial strict inverse semigroup can be so constructed.

The set $X$ in the construction above is the structure set of $S=\left(X ; I_{\alpha}, f_{\alpha, \beta}\right)$. The partially ordered set $X$ in fact is the partially ordered set $S / \mathscr{J}$ of all $\mathscr{J}$-classes of $S=\left(X ; I_{a}, f_{a, \beta}\right)$. In the following "structure set" will stand for "structure set of a combinatorial strict inverse semigroup". Any structure set $X$ satisfies the following (see [1] or [10]).

Proposition 2.2. For any structure set $X$ the following assertions hold: 
(1) $X$ is downwards directed,

(2) for any two elements $\alpha, \beta \in X$ having a common upper bound $\gamma \geqq \alpha, \beta$, the greatest lower bound inf $\{\alpha, \beta\}$ exists in $X$.

The following definitions and results are from [1] where congruences on strict regular semigroups (that is, regular subdirect products of completely simple and/or completely 0 -simple semigroups) are studied.

Definition 1. Let $S=\left(X ; I_{\alpha}, f_{\alpha, \beta}\right)$ be a combinatorial strict inverse semigroup and $\rho \in \mathscr{C}(S)$. For $\alpha, \beta \in X$ put

$$
\alpha \rho, \beta \Leftrightarrow(\exists \gamma \leqq \alpha, \beta)\left(\forall x \in I_{\alpha}^{*}, y \in I_{\beta}^{*}\right) x \rho x f_{\alpha, \gamma}, y \rho y f_{\beta, \gamma} .
$$

An equivalent definition of $\rho_{g}$ is:

$$
\alpha \rho, \beta \Leftrightarrow\left(\exists \gamma \leqq \alpha, \beta, x \in I_{\alpha}^{*}, y \in I_{\beta}^{*}, u, v \in I_{\gamma}^{*}\right) x \rho u, y \rho v \text {. }
$$

For $\alpha \in X$ let $(\alpha]=\{\gamma \in X \mid \gamma \leqq \alpha\}$ denote the principal order ideal in $X$ generated by $\alpha$. By Proposition 2.3 in [1] we have the following.

Proposition 2.3. The relation $\rho_{g}$ is an equivalence relation on $X$ satisfying:

(1) if $\alpha \rho, \beta$ then $\alpha \rho, \gamma \rho, \beta$ for some $\gamma \leqq \alpha, \beta$,

(2) $\rho \mid(\alpha]$ is a semilattice congruence on $(\alpha]$ for each $\alpha \in X$.

Definition 2. Let $X$ be a structure set. An equivalence relation $\xi$ on $X$ is a congruence on $X$ if it satisfies the conditions (1) and (2) of Proposition 2.3. The set of all such congruences on $X$, ordered by inclusion, will be denoted by $\mathscr{C}(X)$.

The following is also from [1] (Corollaries 3.2 and 3.4 and the observation before Corollary 5.14).

Theorem 2.4. Let $S=\left(X ; I_{\alpha}, f_{\alpha, \beta}\right)$ be a combinatorial strict inverse semigroup. Then the following assertions hold.

(1) $\mathscr{C}(X)$ is a complete lattice and a complete $\bigvee$-subsemilattice of $\mathscr{E}(X)$.

(2) The mapping, $; \rho \mapsto \rho$, is an isomorphism between the lattices $(\mathscr{C}(S), \bigcap, \bigvee)$ and $(\mathscr{B}(X), \bigwedge, \bigvee)$

Hence, on a combinatorial strict inverse semigroup $S=\left(X ; I_{\alpha}, f_{\alpha, \beta}\right)$, any congruence $\rho$ is uniquely determined by the relation $\rho_{g}$. This will be essentially used in the following. Notice that $\mathscr{E}(X)$ in general is not a $n$-subsemilattice of $\mathscr{E}(X)$.

3. Atoms and coatoms A congruence $\rho$ covering the identical relation $\varepsilon$ in $\mathscr{C}(S)$ is an 
atom. Dually, each congruence $\rho$ covered by the universal relation $\omega$ is a coatom. Since for $S=\left(X ; I_{\alpha}, f_{\alpha, \beta}\right), \mathscr{C}(S)$ and $\mathscr{C}(X)$ are isomorphic, a congruence $\rho$ is a (co)atom in $\mathscr{C}(S)$ if and only if $\rho$, is a (co)atom in $\mathscr{C}(X)$. We start with coatoms. The following is a reformulation of the analogue for semilattices obtained by Papert [11].

Definition 3. Let $X$ be a structure set. A non empty subset $F \subseteq X$ is a $c$-filter if:

(1) $F$ is an order filter in $X$, that is, $\alpha \in F$ implies $\beta \in F$ for each $\beta \geqq \alpha$,

(2) $F$ is downwards directed, that is, for any $\alpha, \beta \in F$ there is some $\gamma \in F$ such that $\gamma \leqq \alpha, \beta$.

Definition 4. Let $X$ be a structure set and $F \subseteq X$ be a $c$-filter. For $\alpha, \beta \in X$ let

$$
\alpha \rho_{F} \beta \Leftrightarrow \alpha, \beta \in F \text { or } \alpha, \beta \notin F
$$

That is, $\rho_{F}=F \times F \cup X \backslash F \times X \backslash F$.

It is easy to prove that $\rho_{F}$ is a congruence on $X$ for each $c$-filter $F$.

Theorem 3.1. Let $X$ be a structure set. Then the following assertions hold.

(1) A congruence $\rho$ on $X$ is a coatom if and only if $\rho=\rho_{F}$ for some c-filter $F \neq X$.

(2) Each non universal congruence $\rho$ on $X$ is the intersection of some (non empty) set of coatoms. In particular, each non universal congruence $\rho$ is contained in some coatom.

Proof. (1) For each $c$-filter $F \neq X, \rho_{F}$ is a congruence on $X$ and clearly is a coatom since there are precisely two $\rho_{F}$-classes. Conversely, let $\rho$ be a non universal congruence on $X$. At most one $\rho$-class is an ideal in $X$. If $\alpha \rho$ is an ideal then for any $\beta \in X$ there is $\gamma \in X, \gamma \leqq \alpha, \beta$. If $\beta \rho$ is also an ideal then $\gamma \in \alpha \rho \cap \beta \rho$ and therefore $\beta \rho \gamma \rho \alpha$. Let $\alpha \rho$ be a $\rho$-class not being an ideal and let

$$
F=F(\alpha \rho)=\{\beta \in X \mid \beta \geqq \gamma \text { for some } \gamma \in \alpha \rho\}
$$

It is easy to show that $F$ is a $c$-filter. Since $\alpha \rho$ is not an ideal there are $\beta \in \alpha \rho, \gamma \in X \backslash \alpha \rho$ such that $\gamma<\beta$. Then $\gamma \notin F$ for if there would be a $\delta \in \alpha \rho$ such that $\delta \leqq \gamma$ then $\delta \rho \beta$ and $\delta \leqq \gamma<\beta$ imply $\alpha \rho \delta \rho \gamma$ since $\rho \mid(\beta]$ is a congruence on the semilattice $(\beta]$. Consequently, $F \neq X$ and $\rho_{F}$ is a coatom. Let $\beta \in F, \gamma \in X \backslash F$. If $\beta \rho \gamma$ then there is $\delta \leqq \beta, \gamma$ such that $\beta \rho \delta \rho \gamma$. Now there is $\varepsilon \in \alpha \rho, \varepsilon \leqq \beta$. Since $\rho \mid(\beta]$ is a semilattice congruence we observe $\varepsilon=\beta \wedge \varepsilon \rho \delta \wedge \varepsilon$ and thus $\delta \wedge \varepsilon \in \alpha \rho$. Since $\gamma \geqq \delta \geqq \delta \wedge \varepsilon$ we infer that $\gamma \in F$, a contradiction. Consequently, $\rho \subseteq \rho_{F}$. Hence, if we assume in addition that $\rho$ is a coatom then $\rho=\rho_{F}$.

(2) Let $\rho$ be a non universal congruence. Then clearly $\rho \subseteq \bigcap\left\{\rho_{F} \mid \rho \subseteq \rho_{F}\right\}$ and therefore also $\rho \subseteq \bigwedge\left\{\rho_{F} \mid \rho \subseteq \rho_{F}\right\}$. Note that by the proof of (1), the set $\left\{\rho_{F} \mid \rho \subseteq \rho_{F}\right\}$ is not empty. 
We only have to show that for any non $\rho$-related elements $\alpha, \beta \in X$ there is some $\rho_{F}$ containing $\rho$ and separating $\alpha$ and $\beta$. Let $\alpha, \beta \in X$ be such that $(\alpha, \beta) \notin \rho$. Suppose there are $\gamma \in \alpha \rho, v \in \beta \rho$ such that $\gamma \geqq v$. Let $\gamma^{\prime} \in \alpha \rho$; then there is $\delta \leqq \gamma, \gamma^{\prime}$ such that $\delta \in \alpha \rho$. Now $\rho \mid(\gamma]$ is a semilattice congruence so that $\gamma \rho \delta$ implies $v=\gamma \wedge v \rho \delta \wedge v \leqq \gamma^{\prime}$. Therefore, either $\alpha \rho \subseteq F(\beta \rho)$ or $\alpha \rho \cap F(\beta \rho)=\emptyset$. If there are $\gamma, \delta \in \alpha \rho, \mu, v \in \beta \rho$ such that $\gamma \geqq \mu$ and $v \geqq \delta$ then by [1, Lemma 2.6.(2)] we observe that $\alpha \rho=\beta \rho$. Hence if $\alpha \rho \neq \beta \rho$ then $\alpha \rho \cap F(\beta \rho)=\emptyset$ or $\beta \rho \cap F(\alpha \rho)=\emptyset$ (or both) hold. Consequently, the elements $\alpha$ and $\beta$ are separated either by $\rho_{F(\alpha \rho)}$ or by $\rho_{F(\beta \rho)}$ and thus $\rho=\bigcap\left\{\rho_{F(\alpha \rho)} \mid \alpha \rho \in X / \rho\right\}=\bigwedge\left\{\rho_{F(\alpha \rho)} \mid \alpha \rho \in X / \rho\right\}$.

From the description of the coatom $\rho_{F}$ in $\mathscr{C}(X)$ one can easily reconstruct the corresponding coatom $\theta \in \mathscr{C}(S)$ for which $\theta_{g}=\rho_{F}$. A congruence $\rho$ on $X$ is a coatom if and only if there are precisely two $\rho$-classes. This is not true for the coatoms in $\mathscr{C}(S)$. A trivial example is any combinatorial Brandt semigroup $S$ having more than two elements. The corresponding structure set is the two element chain. The only coatom in $\mathscr{C}(S)$ is the identical relation $\varepsilon_{S}$ which has exactly $|S|$ classes. Less trivial examples can be provided easily.

Next we give a description of the atoms in $\mathscr{C}(X)$. It is similar to Hall's description [5] of the atoms in the congruence lattice of a semilattice.

Theorem 3.2. Let $X$ be a structure set, let $\alpha, \beta \in X$ be such that $\beta<\alpha$ and if $\gamma<\alpha$ for some $\gamma \in X$ then $\gamma \leqq \beta$. Define an equivalence relation $\theta(\alpha, \beta)$ on $X$ by

$$
\gamma \theta(\alpha, \beta) \delta \Leftrightarrow\{\gamma, \delta\} \subseteq\{\alpha, \beta\} \text { or } \gamma=\delta .
$$

Then $\theta(\alpha, \beta)$ is an atom in $\mathscr{C}(X)$. Conversely, every atom in $\mathscr{C}(X)$ can be so constructed.

Proof. Let $\alpha, \beta \in X$ be as in the theorem. Then $\theta(\alpha, \beta)$ is obviously an atom in $\mathscr{E}(X)$. Let $\gamma \in X$. Then $\theta(\alpha, \beta) \mid(\gamma]=\varepsilon_{(\gamma]}$ or $\theta(\alpha, \beta) \mid(\gamma]$ is an atom in $\mathscr{C}((\gamma])$ by Hall [5] and thus $\theta(\alpha, \beta) \mid(\gamma]$ is a congruence on $(\gamma]$. Hence $\theta(\alpha, \beta)$ is a congruence on $X$ and thus is an atom in $\mathscr{C}(X)$. Conversely, let $\rho$ be an atom in $\mathscr{C}(X)$. Since $\rho \neq \varepsilon_{X}$ there are $\alpha, \beta \in X, \beta<\alpha$ such that $\alpha \rho \beta$. Let $\chi_{(\alpha)}$ denote the "Rees congruence" with respect to the principal ideal $(\alpha]$. That is,

$$
\gamma \chi_{(a]} \delta \Leftrightarrow \gamma=\delta \text { or } \gamma, \delta \in(\alpha] .
$$

It is easily verified that $\chi_{(a]}$ and $\rho \cap \chi_{(a)}$ are congruences on $X$. In particular, $\rho \cap \chi_{(a)}=\rho \wedge \chi_{(a)}$. Since $\varepsilon \neq \rho \cap \chi_{(a)}$ and $\rho$ is an atom we observe that $\rho \subseteq \chi_{(a)}$. Hence $\rho=\rho \mid(\alpha] \cup \varepsilon_{X}$. Now $\rho \mid(\alpha]$ is an atom in $\mathscr{C}((\alpha])$ for if there is some $\tau \in \mathscr{C}((\alpha])$ such that $\tau \neq \varepsilon_{(a]}$ and $\tau$ is strictly contained in $\rho \mid(\alpha]$ then $\tau \cup \varepsilon_{X}$ is a non identical congruence on $X$, strictly contained in $\rho$. By Hall's characterization of the atoms in $\mathscr{C}((\alpha]), \rho \mid(\alpha]$ admits the description as $\rho \mid(\alpha]=\theta^{(\alpha)}(\alpha, \beta)$ (the upper index denoting the domain of the relation under consideration). Since $\rho=\rho \mid(\alpha] \cup \varepsilon_{X}=\theta^{(\alpha]}(\alpha, \beta) \cup \varepsilon_{X}=\theta^{X}(\alpha, \beta)$ the assertion follows. 
Reconstructing the corresponding atom in $\mathscr{C}(S)$ we obtain the following description.

Corollary 3.3. Let $S=\left(X ; I_{\alpha}, f_{\alpha, \beta}\right)$ be a combinatorial strict inverse semigroup. Then $\rho \in \mathscr{C}(S)$ is an atom if and only if there are $\alpha, \beta \in X, \beta<\alpha$ such that $\gamma<\alpha$ implies $\gamma \leqq \beta$ and

$$
\rho=\left(f_{\alpha, \beta} \cup \varepsilon_{S}\right)\left(f_{\alpha, \beta}^{-1} \cup \varepsilon_{S}\right)
$$

(the product denoting the usual composition of binary relations on S). That is, for $x \in I_{y}^{*}, y \in I_{\delta}^{*}$ we have $x \rho y$ if and only if one of the following holds

(1) $x=y$

(2) $\gamma=\alpha, \delta=\beta$ and $y=x f_{a, \beta}$

(3) $\delta=\alpha, \gamma=\beta$ and $x=y f_{\alpha, \beta}$

(4) $\gamma=\delta=\alpha$ and $x f_{\alpha, \beta}=y f_{\alpha, \beta}$.

A congruence $\rho$ on the structure set $X$ is an atom if and only if $\rho$ has precisely one class containing two elements, the other $\rho$-classes being singletons. Similarly as for the case of coatoms the analogue does not hold for the corresponding atoms in $\mathscr{C}(S)$. A trivial example is again any combinatorial Brandt semigroup containing more than two elements, and less trivial examples can be provided easily.

\section{A subdirect decomposition of $\mathscr{C}(S)$}

For the combinatorial strict inverse semigroup $S$ we provide a decomposition of $\mathscr{C}(S)$ into a subdirect product of congruence lattices of semilattices. As in the previous section, we consider the structure set $X$ of $S$ as a partial semilattice by setting $\alpha \wedge \beta=\inf \{\alpha, \beta\}$, provided this greatest lower bound exists.

Definition 4. A subset $Z \subseteq X$ of a structure set $X$ is a subsemilattice of $X$ if for any $\alpha, \beta \in Z$, their infimum in $X \inf _{X}\{\alpha, \beta\}$ exists and is contained in $Z$. That is, $Z$ is a subsemilattice of $X$ if the partial operation $\wedge$, when restricted to $Z$, provides a total operation on $Z$.

Remark. A subset $Z \subseteq X$, endowed with the induced partial order may be a semilattice but not a subsemilattice of $X$.

Let $X$ be a structure set. By Proposition 2.3, each principal ideal $(\alpha]$ in $X$ is a subsemilattice of $X$. A subsemilattice $Y$ of $X$ is a maximal subsemilattice if there is no subsemilattice $A$ of $X$ strictly containing $Y$.

Lemma 4.1. Each maximal subsemilattice $Y$ of $X$ is an (order) ideal of $X$.

Proof. Let $Z$ be a subsemilattice of $X$ and let $\alpha \in Z$. Let $\beta \in Z$ and $\gamma \in(\alpha]$. Since $Z$ is a subsemilattice, $\inf \{\alpha, \beta\}$ exists and is contained in $(\alpha]$. Since $(\alpha]$ is a subsemilattice, 
$\inf \{\gamma, \inf \{\alpha, \beta\}\}=\inf \{\alpha, \beta, \gamma\}=\inf \{\beta, \gamma\}$ exists and is contained in $(\alpha]$. Consequently, $Z \cup(\alpha]$ is a subsemilattice of $X$ for any $\alpha \in Z$. We infer that for any maximal subsemilattice $Y$ of $X, Y=\bigcup_{a \in Y}(\alpha]$.

The existence of maximal subsemilattices of $X$ follows by a usual Zorn's Lemma argument. Denote by $\mathscr{Y}(X)=\left\{Y_{i} \mid i \in I\right\}$ the collection of all maximal subsemilattices of $X$.

Lemma 4.2. Let $\rho$ be a congruence on the structure set $X$ and $Y$ be a subsemilattice of $X$. Then $\rho \mid Y$ is a semilattice congruence on $Y$.

Proof. Let $\alpha, \beta, \gamma \in Y$ and $\alpha \rho \beta$. By hypothesis, $\alpha \wedge \beta$ exists. There is some $\delta \leqq \alpha, \beta$ such that $\alpha \rho \delta \rho \beta$. Since $\rho \mid(\alpha]$ is a semilattice congruence on $(\alpha]$ we have $\alpha \wedge \beta=\alpha \wedge(\alpha \wedge \beta) \rho \delta \wedge(\alpha \wedge \beta)=\delta$. We infer that $\alpha \rho \alpha \wedge \beta \rho \beta$. The meet $\alpha \wedge \gamma$ exists since $Y$ is a subsemilattice of $X$. Hence $\alpha \wedge \gamma=\alpha \wedge(\alpha \wedge \gamma) \rho(\alpha \wedge \beta) \wedge(\alpha \wedge \gamma)=$ inf $\{\alpha, \beta, \gamma\}$ and dually also $\beta \wedge \gamma \rho \inf \{\alpha, \beta, \gamma\}$ so that $\alpha \wedge \gamma \rho \beta \wedge \gamma$.

Corollary 4.3. If $X$ is a semilattice then the congruences on $X$ are precisely the semilattice congruences.

We now are able to prove the main result. The idea is similar to Hamilton's idea to decompose the congruence lattice of a tree into a subdirect product of the congruence lattices of the maximal subchains (see [6]).

Theorem 4.4. Let $X$ be the structure set of a combinatorial strict inverse semigroup and let $\left\{Y_{i} \mid i \in I\right\}$ be the collection of all maximal subsemilattices of $X$. Then the mapping

$$
\mathscr{C}(X) \rightarrow \prod_{i \in I} \mathscr{C}\left(Y_{i}\right), \quad \rho \mapsto\left(\rho \mid Y_{i}\right)_{i \in I}
$$

is a lattice isomorphism between $\mathscr{C}(X)$ and the complete subdirect product

$$
\Phi=\left\{\left(\rho_{i}\right)_{i \in I} \in \prod_{i \in I} \mathscr{C}\left(Y_{i}\right)\left|\rho_{i}\right| Y_{i} \cap Y_{j}=\rho_{j} \mid Y_{i} \cap Y_{j}, i, j \in I\right\}
$$

Proof. Let $i \in I$ be a fixed element and let $\left\{p_{k} \mid k \in K\right\}$ be a collection of congruences on $X$. By Theorem 2.4 it follows that $\bigwedge_{k \in K} \rho_{k}=\left(\bigcap_{k \in K} \rho_{k}\right)^{0}$, that is, the greatest congruence on $X$ contained in $\bigcap_{k \in K} \rho_{k}$. In the following we shall omit the subscript "k,K". First we observe that $\left(\bigwedge \rho_{k}\right)\left|Y_{i} \subseteq\left(\bigcap \rho_{k}\right)\right| Y_{i}=\bigcap \rho_{k} \mid Y_{i}$. For each $k$ put $\rho_{k}^{\prime}=$ $\rho_{k} \mid Y_{i} \cup \varepsilon_{X}$. Then $\rho_{k}^{\prime}$ is a congruence on $X$ and $\rho_{k}^{\prime} \subseteq \rho_{k}$. Now $\bigcap \rho_{k}^{\prime}=\bigcap\left(\rho_{k} \mid Y_{i} \cup \varepsilon_{X}\right)=$ $\left(\bigcap \rho_{k} \mid Y_{i}\right) \cup \varepsilon_{X}$ which is a congruence on $X$. Hence $\bigwedge \rho_{k}^{\prime}=\bigcap \rho_{k}^{\prime} \subseteq \bigwedge \rho_{k}$ since $\rho_{k}^{\prime} \subseteq \rho_{k}$ for all $k$. Restricting to $Y_{i}$ we get $\left(\bigwedge \rho_{k}^{\prime}\right)\left|Y_{i}=\left(\bigcap \rho_{k}^{\prime}\right)\right| Y_{i}=\left(\bigcap \rho_{k} \mid Y_{i} \cup \varepsilon_{X}\right)\left|Y_{i}=\bigcap \rho_{k}\right| Y_{i}$ and hence $\bigcap \rho_{k}\left|Y_{i} \subseteq\left(\bigwedge \rho_{k}\right)\right| Y_{i}$. Consequently, $\left(\bigwedge \rho_{k}\right)\left|Y_{i}=\bigcap \rho_{k}\right| Y_{i}$ and thus the mapping 


$$
\mathscr{C}(X) \rightarrow \mathscr{C}\left(Y_{i}\right), \quad \rho \mapsto \rho \mid Y_{i}
$$

is a complete $\bigwedge$-homomorphism. For the join we observe that the inclusion $\bigvee \rho_{k} \mid Y_{i} \subseteq$ $\left(\bigvee \rho_{k}\right) \mid Y_{i}$ is trivial. To show the converse, let $\alpha, \beta \in Y_{i}$ be such that $\alpha \bigvee \rho_{k} \beta$. There exist $\alpha_{0}, \ldots, \alpha_{n} \in X$ and $\rho_{1}, \ldots, \rho_{n} \in\left\{\rho_{k} \mid k \in K\right\}$ such that $\alpha=\alpha_{0} \rho_{1} \alpha_{1} \ldots \alpha_{n-1} \rho_{n} \alpha_{n}=\beta$. For each $j$, $1 \leqq j \leqq n$ there is $\beta_{j} \leqq \alpha_{j-1}, \alpha_{j}$ such that $\alpha_{j-1} \rho_{j} \beta_{j} \rho_{j} \alpha_{j}$. Since $\beta_{1}, \beta_{2} \leqq \alpha_{1}$, the meet $\gamma_{2}=\inf \left\{\beta_{1}, \beta_{2}\right\}$ exists. By induction, $\gamma_{j}=\gamma_{j-1} \wedge \beta_{j}=\inf \left\{\beta_{1}, \beta_{2} \ldots \beta_{j}\right\}$ exists and $\gamma_{j} \leqq$ $\alpha_{0}, \ldots, \alpha_{j}$. The relation $\alpha_{j} \rho_{j+1} \beta_{j+1}$ yields $\gamma_{j}=\alpha_{j} \wedge \gamma_{j} \rho_{j+1} \beta_{j+1} \wedge \gamma_{j}=\gamma_{j+1}$. Since the maximal subsemilattice $Y_{i}$ is an ideal (Lemma 4.1), $\gamma_{j} \in Y_{i}$ for all $j$. Put $\gamma_{n}=\gamma$ then $\gamma \leqq \alpha, \beta$ and $\alpha \bigvee \rho_{k} \mid Y_{i} \gamma$. By the same argument there is some $\delta \leqq \alpha, \beta$ such that $\beta \bigvee \rho_{k} \mid Y_{i} \delta$. Now $\alpha \geqq \alpha \wedge \beta \geqq \gamma$ and $\beta \geqq \alpha \wedge \beta \geqq \delta$ imply $\alpha \bigvee \rho_{k}\left|Y_{i} \alpha \wedge \beta \bigvee \rho_{k}\right| Y_{i} \beta$. In particular, $\bigvee \rho_{k} \mid Y_{i}=$ $\left(\bigvee \rho_{k}\right) \mid Y_{i}$ and thus the mapping

$$
\mathscr{C}(X) \rightarrow \mathscr{C}\left(Y_{i}\right), \quad \rho \mapsto \rho \mid Y_{i}
$$

is a complete $\bigvee$-homomorphism. For $\rho_{i} \in \mathscr{C}\left(Y_{i}\right)$ the relation $\rho=\rho_{i} \cup \varepsilon_{X}$ is a congruence on $X$ such that $\rho \mid Y_{i}=\rho_{i}$. Hence the homomorphism $\rho \mapsto \rho \mid Y_{i}$ is surjective. Now let $\rho, \eta \in \mathscr{C}(X)$ be such that $\rho \neq \eta$. We may assume that there are $\alpha, \beta \in X$ such that $\alpha \rho \beta$ and $(\alpha, \beta) \notin \eta$. By a Zorn's Lemma argument there are maximal subsemilattices $Y_{i}, Y_{j}$ of $X$ such that $(\alpha] \subseteq Y_{i}$ and $(\beta] \subseteq Y_{j}$. Now there is $\gamma \leqq \alpha, \beta$ such that $\alpha \rho \gamma \rho \beta$. Then either $(\alpha, \gamma) \notin \eta$ or $(\beta, \gamma) \notin \eta$ and hence either $\rho\left|Y_{i} \neq \eta\right| Y_{i}$ or $\rho\left|Y_{j} \neq \eta\right| Y_{j}$. It follows that the mapping $\mathscr{C}(X) \rightarrow \prod \mathscr{C}\left(Y_{i}\right), \rho \mapsto\left(\rho \mid Y_{i}\right)_{i \in I}$ is injective. Finally, let $\left(\rho_{i}\right) \in \prod \mathscr{C}\left(Y_{i}\right)$ be such that for all $i, j \in I, \rho_{i}\left|Y_{i} \cap Y_{j}=\rho_{j}\right| Y_{i} \cap Y_{j}$. Put $\rho_{i}^{\prime}=\rho_{i} \cup \varepsilon_{X}$. Then $\rho_{i}^{\prime} \in \mathscr{C}(X)$ for each $i \in I$. Let $\rho=\bigvee \rho_{i}^{\prime}$. We obviously have $\rho_{i} \subseteq \rho \mid Y_{i}$. Choose a fixed $j \in I$ and let $\alpha, \beta \in Y_{j}$ be such $\alpha \rho \beta$. There are $\alpha_{0}, \ldots, \alpha_{n} \in X$ and $\rho_{1}^{\prime}, \ldots, \rho_{n}^{\prime} \in\left\{\rho_{i}^{\prime} \mid i \in I\right\}$ such that $\alpha=\alpha_{0} \rho_{1}^{\prime} \alpha_{1} \ldots \alpha_{n-1} \rho_{n}^{\prime} \alpha_{n}=\beta$. Similarly as above there are $\beta_{1}, \ldots, \beta_{n} \in X$ such that $\alpha \geqq \beta_{1} \geqq \cdots \geqq \beta_{n} \leqq \beta$ and $\beta_{k-1} \rho_{k}^{\prime} \beta_{k}$. Since $(\alpha] \subseteq Y_{j}$ we have $\beta_{k} \in Y_{j}$ for all $k$. Since $\rho_{k}^{\prime}\left|Y_{j} \cap Y_{k}=\rho_{j}^{\prime}\right| Y_{j} \cap Y_{k}=\rho_{j} \mid Y_{j} \cap Y_{k}$ we obtain $\beta_{k-1} \rho_{j} \beta_{k}$ for all $k$ and thus $\alpha \rho_{j} \beta_{n}$, that is, $\alpha \rho_{j} \gamma$ for some $\gamma \leqq \alpha, \beta$. Dually also $\beta \rho_{j} \delta$ for some $\delta \leqq \alpha, \beta$, and therefore, as above, $\alpha \rho_{j} \alpha \wedge \beta \rho_{j} \beta$. Consequently, $\rho \mid Y_{j} \subseteq \rho_{j}$ and thus $\rho \mid Y_{j}=\rho_{j}$. This holds for each $j \in I$ and thus the proof is complete.

We have thus shown that the congruence lattice of a combinatorial strict inverse semigroup is a subdirect product of congruence lattices of semilattices.

Definition 5. A (complete) lattice $L$ is

(1) completely semidistributive if for any $\sigma, \rho_{i} \in L, i \in I$ such that $\rho_{i} \wedge \sigma=\rho_{j} \wedge \sigma$ for all $i, j \in I$, then $\left(\bigvee \rho_{i}\right) \wedge \sigma=\bigvee\left(\rho_{i} \wedge \sigma\right)$.

(2) $M$-symmetric if the modularity relation $M$ on $L$, defined by $\rho M \sigma$ if and only if $\tau=(\tau \vee \rho) \wedge \sigma$ for all $\tau \in[\rho \wedge \sigma, \sigma]$, is a symmetric relation.

It has been shown by Papert [11] that the congruence lattice of any semilattice is completely semidistributive, and by Jones [9, Theorem 3.3] that the congruence lattice 
of a semilattice is $M$-symmetric. By Jones [8, Proposition 5.1], $M$-symmetry is preserved by subdirect products. Theorem 4.4 therefore yields the following:

Corollary 4.5. Let $T \in \mathscr{B}$. Then the following assertions hold:

(1) $\mathscr{C}(T)$ is completely semidistributive,

(2) $\mathscr{C}(T)$ is $M$-symmetric.

Furthermore, the classes $\mathscr{C}(\mathscr{B})$ and $\mathscr{C}(\mathscr{S})$ generate the same quasivariety of lattices.

Notice that $M$-symmetry of $\mathscr{C}(T)$ also follows from the result of Jones [9, Theorem 3.3] by taking into account that for any $\rho, \eta \in \mathscr{C}(T), \rho=\eta$ if and only $\rho|E=\eta| E, E$ denoting the set of idempotents of $T$.

\section{Weak relative pseudocomplements and pseudocomplements}

Let $L$ be a lattice, $\rho, \sigma \in L, \rho \leqq \sigma$. If there is a greatest element $\tau \in L$ such that $\tau \wedge \sigma=\rho$ then $\tau$ is a weak relative pseudocomplement of $\rho$ and $\sigma$, to be denoted by $\sigma * \rho$. If $\sigma * \rho$ exists for all pairs $\rho \leqq \sigma$ in $L$ then $L$ is weakly relatively pseudocomplemented. If $L$ has a least element 0 then for any $\sigma \in L$ the weak relative pseudocomplement $\sigma * 0$ is called the pseudocomplement of $\sigma$ (provided it exists). An important result of Papert [11] asserts that the congruence lattice $\mathscr{E}(Y)$ of any semilattice $Y$ is weakly relatively pseudocomplemented and hence also is pseudocomplemented. We shall extend this result to congruence lattices of combinatorial strict inverse semigroups. As in the previous section, for a combinatorial strict inverse semigroup $S$, the congruence lattice $\mathscr{C}(S)$ will be realized as the lattice $\mathscr{C}(X)$ of all congruences on the structure set $X$ of $S$ and thus as a subdirect product of the congruence lattices of the maximal subsemilattices $Y_{i}$ of $X$ as described in Theorem 4.4 .

Let $X$ be a structure set, $\mathscr{Y}(X)=\left\{Y_{i} \mid i \in I\right\}$ be the collection of all maximal subsemilattices of $X$. For each $\rho \in \mathscr{C}(X), i \in I$ let $\rho_{i}=\rho \mid Y_{i} \in \mathscr{C}\left(Y_{i}\right)$. Let $\rho, \sigma \in \mathscr{C}(X), \rho \subseteq \sigma$. Then $\rho_{i} \subseteq \sigma_{i}$ for all $i \in I$. Further, if $\tau \cap \sigma=\rho$ for some $\tau \in \mathscr{C}(X)$ then $\tau_{i} \cap \sigma_{i}=\rho_{i}$ for each $i$ and hence $\tau_{i} \subseteq \sigma_{i} *_{i} \rho_{i}, *_{i}$ denoting the weak relative pseudocomplement in $\mathscr{C}\left(Y_{i}\right)$.

Lemma 5.1. Let $Y$ be a semilattice, $Z$ be an ideal (and thus a subsemilattice) of $Y$, $\rho, \sigma \in C(Y), \rho \subseteq \sigma$. Then $\sigma\left|Z *_{Z} \rho\right| Z=\sigma *_{Y} \rho \mid Z\left(*_{Z}\right.$ respectively $*_{Y}$ denoting weak relative pseudocomplementation in $\mathscr{C}(Z)$ respectively $\mathscr{C}(Y))$.

Proof. Put $\rho^{\prime}=\rho \mid Z$ and $\sigma^{\prime}=\sigma \mid Z$. Then $\rho^{\prime} \subseteq \sigma^{\prime}$ and thus $\sigma^{\prime}{ }_{Z} \rho^{\prime}$ exists. Now $\sigma \cap\left(\sigma *_{Y} \rho\right) \mid Z=\sigma^{\prime} \cap\left(\sigma *_{Y} \rho \mid Z\right)=\varepsilon_{Z}$ so that $\sigma *_{Y} \rho \mid Z \subseteq \sigma^{\prime} *_{Z} \rho^{\prime}$. By [11, Theorem 2], for $\alpha, \beta \in Z$ we have

$$
\alpha \sigma^{\prime} z_{Z} \rho^{\prime} \beta \Leftrightarrow\left((\forall \delta, \gamma \in Z) \delta \sigma^{\prime} \gamma \Rightarrow\left(\alpha \wedge \delta \rho^{\prime} \alpha \wedge \gamma \Leftrightarrow \beta \wedge \delta \rho^{\prime} \beta \wedge \gamma\right)\right) .
$$

We intend to prove that $\alpha \sigma *_{Y} \rho \beta$ whenever $\alpha \sigma^{\prime} z_{Z} \rho^{\prime} \beta$. Let $\alpha, \beta \in Z$ and $\alpha \sigma^{\prime} z_{Z} \rho^{\prime} \beta$. We 
have to prove that $\alpha \sigma *_{Y} \rho \beta$, that is, $\alpha \wedge \delta \rho \alpha \wedge \gamma \Leftrightarrow \beta \wedge \delta \rho \beta \wedge \gamma$ for any $\gamma, \delta \in Y$ such that $\delta \sigma \gamma$. Let $\gamma, \delta \in Y$ be such that $\delta \sigma \gamma$. Since $Z$ is an ideal, $\alpha \wedge \delta, \alpha \wedge \gamma, \beta \wedge \delta$, $\beta \wedge \gamma \in Z$, and $\alpha \wedge \delta \sigma^{\prime} \alpha \wedge \gamma, \beta \wedge \delta \sigma^{\prime} \beta \wedge \gamma$. By $\alpha \sigma^{\prime} *_{Z} \rho^{\prime} \beta$ we have

$$
\alpha \wedge \delta=\alpha \wedge(\alpha \wedge \delta) \rho^{\prime} \alpha \wedge(\alpha \wedge \gamma)=\alpha \wedge \gamma \Leftrightarrow \beta \wedge(\alpha \wedge \delta) \rho^{\prime} \beta \wedge(\alpha \wedge \gamma)
$$

and

$$
\beta \wedge \delta=\beta \wedge(\beta \wedge \delta) \rho^{\prime} \beta \wedge(\beta \wedge \gamma)=\beta \wedge \gamma \Leftrightarrow \alpha \wedge(\beta \wedge \delta) \rho^{\prime} \alpha \wedge(\beta \wedge \gamma) .
$$

Since $\alpha \wedge(\beta \wedge \delta)=\beta \wedge(\alpha \wedge \delta)$ and $\alpha \wedge(\beta \wedge \gamma)=\beta \wedge(\alpha \wedge \gamma)$ we infer that $\alpha \wedge \delta \rho^{\prime} \alpha \wedge$ $\gamma \Leftrightarrow \beta \wedge \delta \rho^{\prime} \beta \wedge \gamma$, that is, $\alpha \wedge \delta \rho \alpha \wedge \gamma \Leftrightarrow \beta \wedge \delta \rho \beta \wedge \gamma$. Consequently, $\alpha \sigma *_{Y} \rho \beta$ and the assertion is proved.

Using Theorem 4.4 it is now easy to obtain the mentioned result.

Theorem 5.2. The congruence lattice of a combinatorial strict inverse semigroup $S$ is isomorphic to a subdirect product of congruence lattices of semilattices closed under componentwise formation of weak relative pseudocomplements and pseudocomplements. In particular, $\mathscr{C}(S)$ is pseudocomplemented.

Proof. Let $X$ be the structure set of $S$ and $\left\{Y_{i} \mid i \in I\right\}$ be the collection of all maximal subsemilattices of $S$. By Theorem 4.4, $\mathscr{C}(X)$ is isomorphic to

$$
\Phi=\left\{\left(\rho_{i}\right)_{i \in I} \in \prod_{i \in I} \mathscr{C}\left(Y_{i}\right)\left|\rho_{i}\right| Y_{i} \cap Y_{j}=\rho_{j} \mid Y_{i} \cap Y_{j}, i, j \in I\right\}
$$

Let $\rho, \sigma \in C(X), \rho \subseteq \sigma$. Then $\rho_{i} \subseteq \sigma_{i}$ and hence $\sigma_{i} *_{i} \rho_{i}$ exists for each $i, *_{i}$ denoting the weak relative pseudocomplements in $\mathscr{C}\left(Y_{i}\right)$. By Lemma 5.1,

$$
\begin{aligned}
\sigma_{i} *_{i} \rho_{i} \mid Y_{i} \cap Y_{j} & =\left(\sigma_{i} \mid Y_{i} \cap Y_{j}\right) *\left(\rho_{i} \mid Y_{i} \cap Y_{j}\right) \\
& =\left(\sigma_{j} \mid Y_{i} \cap Y_{j}\right) *\left(\rho_{j} \mid Y_{i} \cap Y_{j}\right) \\
& =\sigma_{j} *_{j} \rho_{j} \mid Y_{i} \cap Y_{j},
\end{aligned}
$$

* denoting the weak relative pseudocomplement in $\mathscr{C}\left(Y_{i} \cap Y_{j}\right)$. Hence $\left(\sigma_{i} *_{i} \rho_{i}\right)_{i \in I} \in \Phi$ and thus $\sigma * \rho=\bigvee_{i \in I} \sigma_{i} *_{i} \rho_{i} \cup \varepsilon_{X}$ is the weak relative pseudocomplement of $\rho$ and $\sigma$ in $\mathscr{C}(X)$.

\section{An example}

In [1], it is shown that there is a (finite) combinatorial strict inverse semigroup $S$ whose structure set $X$ consists of five elements and is depicted by Fig. 1 . 


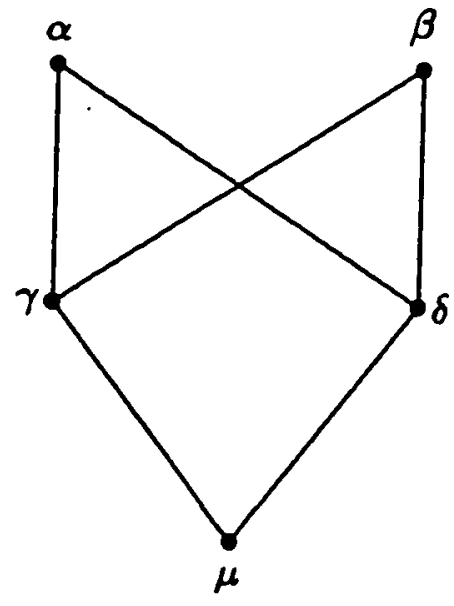

FIGURE 1

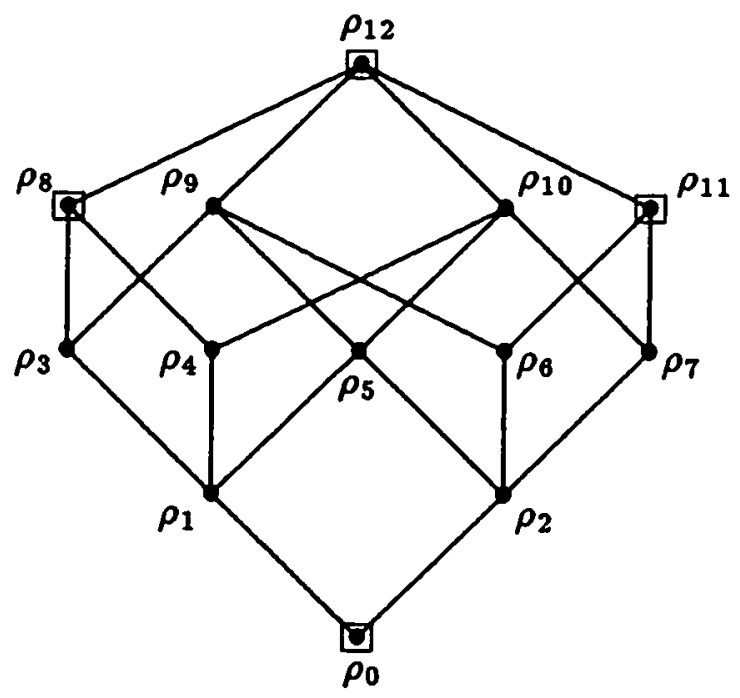

FIGURE 2 
In particular, $X$ is not a semilattice. All congruences on $X$ are listed below (by denoting the corresponding partitions, and only the non singleton classes are mentioned):

$$
\begin{aligned}
& \rho_{0}: \varepsilon_{X} \\
& \rho_{1}:\{\mu, \gamma\} \\
& \rho_{2}:\{\mu, \delta\} \\
& \rho_{3}:\{\mu, \gamma\},\{\delta, \alpha\} \\
& \rho_{4}:\{\mu, \gamma\},\{\delta, \beta\} \\
& \rho_{5}:\{\mu, \gamma, \delta\} \\
& \rho_{6}:\{\mu, \delta\},\{\gamma, \alpha\} \\
& \rho_{7}:\{\mu, \delta\},\{\gamma, \beta\} \\
& \rho_{8}:\{\mu, \gamma\},\{\alpha, \beta, \delta\} \\
& \rho_{9}:\{\mu, \gamma, \alpha, \delta\} \\
& \rho_{10}:\{\mu, \gamma, \beta, \delta\} \\
& \rho_{11}:\{\mu, \delta\},\{\alpha, \beta, \gamma\} \\
& \rho_{12}: \omega_{X} .
\end{aligned}
$$

The lattice $\mathscr{C}(X)$ is depicted by Fig. 2. The Boolean elements in $\mathscr{C}(X)$, that is, those elements $\rho$ in $\mathscr{C}(X)$ which are pseudocomplements of elements of $\mathscr{C}(X)$ are precisely the elements $\rho_{0}, \rho_{8}, \rho_{11}, \rho_{12}$. If $\mathscr{C}(X)$ was isomorphic to the congruence lattice of some semilattice $Y$ then by [11, Theorem 5], $Y$ could be embedded into the (lattice of the) Boolean elements of $\mathscr{C}(Y) \cong \mathscr{C}(X)$. But no subsemilattice of the four element diamond lattice has a congruence lattice isomorphic to $\mathscr{C}(X)$. Hence $\mathscr{C}(X)$ and thus also $\mathscr{C}(S)$ cannot be isomorphic to the congruence lattice of any semilattice.

\section{REFERENCES}

1. K. Auinger, The congruence lattice of a strict regular semigroup, J. Pure Appl. Algebra 81 (1992), 219-245. $51-58$

2. R. Freese and J. B. Nation, Congruence lattices of semilattices, Pacific J. Math. 49 (1973),

3. R. Freese and J. B. Nation, Congruence lattices of semilattices revisited, preprint.

4. G. GRẢTZR, General Lattice Theory (Birkhäuser, Basel, 1978).

5. T. E. Hall, On the lattice of congruences on a semilattice, J. Austral. Math. Soc. 12 (1971), 456-460.

6. H. B. Hamilton, Semilattices whose structure lattice is distributive, Semigroup Forum 8 (1974), 245-253. 
7. J. M. Howle, An Introduction to Semigroup Theory (Academic Press, London, 1976).

8. P. R. Jones, Semimodular inverse semigroups, J. London Math. Soc. 17 (1978), 446-456.

9. P. R. Jones, On congruence lattices of regular semigroups, J. Algebra 82 (1983), 18-39.

10. K. S. S. NAmbooripad, Pseudo-semilattices and biordered sets III: regular locally testable semigroups, Simon Stevin 56 (1982), 239-256.

11. D. PAPERT, Congruence relations in semilattices, J. London Math. Soc. 39 (1964), 723-729.

12. M. Petrich, Inverse Semigroups (Wiley, New York, 1984).

13. G. I. ZhiтомiRsky, A characterization of congruence lattices of semilattices (Russian), Izv. Vyssh. Ucebn. Zaved. Dep. VINITI (1984).

INSTITUT FOR MATHEMATIK

STRUDLHOFGASSE 4

A-1090 WIEN

Austria

E-mail address: A813IDAT@AWIUNIII.BITNET 